\title{
A Parametric Model Approach for Quantification of Short Term QT Variability Uncorrelated with Heart Rate Variability
}

\author{
R Almeida ${ }^{*}, \mathrm{E} \mathrm{Pueyo}^{+}, \mathrm{JP}_{\mathrm{Martinez}}{ }^{+}, \mathrm{AP} \mathrm{Rocha}^{*}, \mathrm{~S} \mathrm{Olmos}^{+}, \mathrm{P} \mathrm{Laguna}^{+}$ \\ *Dep. de Matemática Aplicada, Faculdade de Ciências, Universidade do Porto, Portugal \\ ${ }^{+}$Comm. Techn. Group, Aragon Institute of Eng. Research, University of Zaragoza, Spain
}

\begin{abstract}
In this work we propose to assess the relation between $H R V$ and QTV measured by an automatic delineator. A low order linear autoregressive model on RR versus $Q T$ interactions was used to explore short term relations and quantify the fractions of QTV correlated and not correlated with HRV. Power spectral density measures were estimated from the total QTV and from the two separated fractions using the proposed model. Simulated $R R$ and $Q T$ series were used to quantify the error bounds associated to the method performance. ECG records of young normal subjects were processed to obtain the RR and $Q T$ series. The high QTV fraction not correlated with $R R$ found in these records (over $40 \%$ in $98 \%$ of the segments) indicates that an important part of QTV can be driven by other factors rather than by heart rate, and may contain complementary information.
\end{abstract}

\section{Introduction}

The duration of ventricular repolarization (VR) expressed by the QT interval is known to be influenced mainly by the RR interval but also by other factors. Moreover, the QT interval presents a beat-to-beat variability (QTV) which presumably will be largely driven by heart rate variability (HRV). However, it has not been yet clearly quantified which fraction of QTV is effectively correlated with HRV.

The uncertainty in $\mathrm{T}$ wave end delineation (both on manual and automatic measures) allied to the smaller amplitude of QTV as compared to HRV, represent main difficulties in exploring this relation and led some authors to look for alternative VR interval measures like the RTapex interval (RT) [1]. Such an approach assumes that the cardiac cycle length dependence of VR is essentially concentrated on the early portion of the QT interval and ignores fluctuations in VR that mainly affect the last part of the $\mathrm{T}$ wave. Furthermore, the shorter length of RTapex interval compared to QT additionally penalises the variability measurements.

An open loop model of the RT-RR variability interactions was proposed by Porta et al [1] taking into account the possible dependency of the RT interval on its past samples and on past values of the RR interval (as shown in recent studies [2]). This linear, dynamic parametric approach allows to separate the fraction of the RT variability driven by RR changes from that uncorrelated with HRV.

A wavelet transform based ECG delineation system [3] has proven to be quite robust against noise and morphological variations. This fact together with the automatic nature of the detector that will avoid the inter/intra-expert variability in the measurements, led us to use the measured intervals to explore the relation between HRV and QTV.

Our proposal is to use a linear low order model approach similar to the proposed in [1] in order to explore the short term HRV and QTV relations and quantify the fraction of QTV not driven by RR.

\section{Methods}

\subsection{Model formulation}

The RR, QT (QRS onset to T wave end) and RTapex intervals were measured using the marks supplied by the automatic delineation [3] and corrected for the mean.

A linear parametric model, based on the one proposed by Porta et al [1], was considered to express RR and QT variability interactions in an open loop model (Fig. 1),

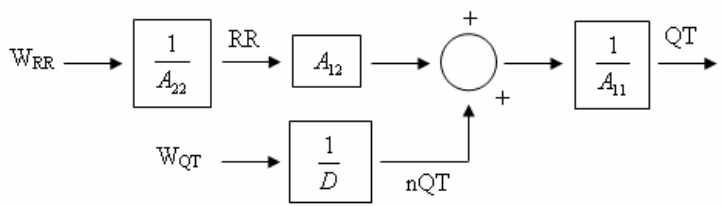

Figure 1. Schematic representation of the QTV versus HRV interactions model.

where $A_{11}, A_{12}, A_{22}$ and $D$ are polynomials in $\mathrm{z}^{-1}$ with coefficients $a_{11}, a_{12}, a_{22}$ and $d$, respectively. $W_{R R}$ and $W_{Q T}$ are uncorrelated white noises with zero mean and variances $\lambda^{2}{ }_{\mathrm{RR}}$ and $\lambda^{2}{ }_{\mathrm{QT}}$. RR trend was modeled as an $\mathrm{AR}$ stationary random process given by,

$$
R R[i]=-\sum_{k=1}^{n} a_{22}[k] R R[i-k]+W_{R R}[i]
$$


where $n$ stands for the model order. QT trend was assumed to result from two uncorrelated sources, one driven by heart rate and the other one uncorrelated,

$$
\begin{aligned}
& Q T[i]=\sum_{k=0}^{n} a_{12}[k] R R[i-k]-\sum_{k=1}^{n} a_{11}[k] Q T[i-k]+n Q T[i] \\
& n Q T[i]=-\sum_{k=1}^{n} d[k] n Q T[i-k]+W_{Q T}[i]
\end{aligned}
$$

As both QT and RR series are unevenly sampled we used the mean RR interval $(\overline{R R})$ as sampling rate for estimating the Power Spectral Density (PSD) functions. For low frequencies far from the Nyquist frequency, this assumption has been shown acceptable [4]. The PSD of QT $\left(S_{Q T}\right)$ can be computed as the sum of the two partial spectra that express the contribution of each source

$$
S_{Q T}(\omega)=S_{Q T / W_{R R}}(\omega)+S_{Q T / W_{Q T}}(\omega)
$$

where

$$
\begin{aligned}
& S_{Q T / W_{R R}}(\omega)=\overline{R R} \lambda_{R R}^{2}\left|\frac{A_{12}(z)}{A_{11}(z) A_{22}(z)}\right|_{z=\exp (j \omega \overline{R R})}^{2} \\
& S_{Q T / W_{Q T}}(\omega)=\overline{R R} \lambda_{Q T}^{2}\left|\frac{1}{A_{11}(z) D(z)}\right|_{z=\exp (j \omega \overline{R R})}^{2}
\end{aligned}
$$

The same approach can be applied over RR-RTapex variability interactions, as already proposed by Porta et al [1] and we used it in this work for comparison.

\subsection{Model identification}

The polynomial $A_{22}$ can be estimated using least squares, while $A_{11}, A_{12}$ and $\mathrm{D}$ estimations are iteratively obtained using a generalized least squares (GLS) methodology [5] applied to the problem of equations (2). For adequate model orders the GLS convergence to white noise residual $W_{Q T}$ is expected to occur in a reasonable small number of iterations; in this implementation a maximum of 100 iterations was allowed.

Even orders from 6 to 18 were considered and the same order was assumed for all polynomials in the model, for simplicity. An order was considered to be adequate for modelling a given segment of data if the normalized autocorrelations and cross-correlation of $W_{R R}$ and $W_{Q T}$ satisfied a $10 \%$ confidence bilateral test both in lags lower than 40 beats and considering all lags.

\subsection{Performance evaluation}

The described methodology was applied both to real data from databases and simulated sequences. The two partial PSD were calculated using the estimated coefficients and equations (4) and (5). The ratios between the measures of the uncorrelated fraction and total QTV allowed to evaluate the relative contribution of the QTV not driven by RR. Low Frequency $(0.04-0.15 \mathrm{~Hz})$ and
High Frequency $(0.15-0.4 \mathrm{~Hz})$ bands were considered and analyzed separately so as to evaluate whether the uncorrelated fraction differed for different frequency bands. Total Power was considered as the band from 0.04 $\mathrm{Hz}$ to the highest frequency present in each spectrum.

\subsubsection{Simulation set-up}

The validation of the model was based on simulated RR and QT series with known QTV fraction correlated with RR. We simulated tachograms using integral pulse frequency modulation model (sampling frequencies of 500 and $1000 \mathrm{~Hz}$ ) with an autogressive (AR) modulating signal [4]. Two models (RRI and RR2) with different main frequency components (Fig. 2) were used to simulate 50 realizations of uncorrelated RR trends.

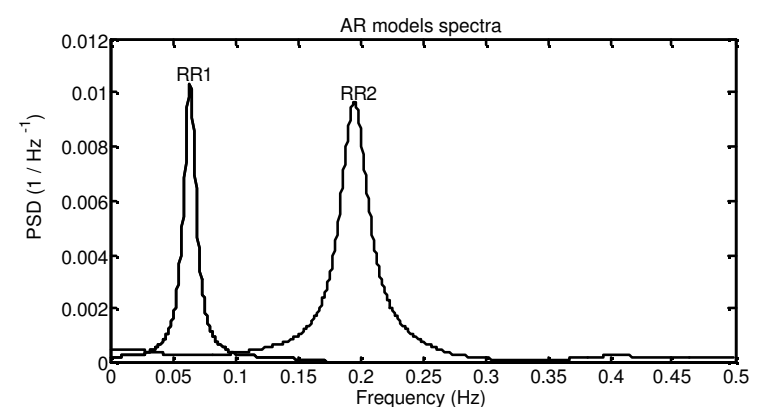

Figure 2. Spectra of the RR1 and RR2 series considered in the simulation.

A constant reference QT value, $q t_{0}$, was used to define the following test data:

A1) $R R=R R 1$ and $Q T=q t_{0} \sqrt{R R 1}$;

B1) $R R=R R 1$ and $Q T=q t_{0} \sqrt{R R 2}$;

C1) $R R=R R 1$ and $Q T=q t_{0} \sqrt{R R 1}+q t_{0} \sqrt{R R 2}-\overline{q t_{0} \sqrt{R R 2}}$;

A2) $R R=R R 2$ and $Q T=q t_{0} \sqrt{R R 2}$;

B2) $R R=R R 2$ and $Q T=q t_{0} \sqrt{R R 1}$;

C2) $R R=R R 2$ and $Q T=q t_{0} \sqrt{R R 2}+q t_{0} \sqrt{R R 1}-\overline{q t_{0} \sqrt{R R 1}}$.

To evaluate the methodology in a more realistic scenario we also produced an artificial ECG. A clean and well defined template beat was interpolated to reflect the QTV characteristics previously defined with the corresponding sampling frequency. The ECG was obtained by concatenation of the modified beats, following the simulated tachogram. To avoid strange morphologies, the interpolation was limited to the interval between the end of the QRS complex and 25 samples prior to the $\mathrm{T}$ wave end. The signals obtained from the series defined in A1, B2, C2, A2, B2 and C2 were processed by the automatic delineation system [3] to obtain the new fiducial marks and the correspondent interval measurements were taken as a second test data denoted by D1, E1, F1, D2, E2 and F2, respectively. 
The RR and QT series were simulated for sampling frequencies of 500 and $1000 \mathrm{~Hz}$ and the measured series were obtained. The ratios $(\%)$ between the measures on uncorrelated fraction and total QTV were calculated in each frequency band for all 12 situations. The errors were calculated as the differences between these values and the reference ratios calculated by using the relation, $Q T-\overline{Q T}=P_{M}(Q T-\overline{Q T})+P_{M} \perp(Q T-\overline{Q T})$, where

$M$ is the subspace generated by the simulated $R R-\overline{R R}$ and all its corresponding delayed vectors up to the highest order (18), $P_{M}(Q T-\overline{Q T})$ and $P_{M} \perp(Q T-\overline{Q T})$ stand for the projections of the simulated $Q T-\overline{Q T}$ over $M$ and its orthogonal subspace, respectively. Thus, the ratios between the variability measures of projection over the subspace M and QT measures represent the QTV fraction correlated with RR and the remaining percentage can be used as reference for the fraction uncorrelated to RR.

\subsubsection{Real data}

ECG recordings of young normal subjects from POLI/MEDLAV and Politecnico Ca' Granda databases [6] were used in this study, consisting of 3 leads sampled at $500 \mathrm{~Hz}$. Each lead was processed by the delineation system in [3].

Anomalies in RR series were identified [7] and QT intervals out of a 3-standard deviation band were rejected as possible outliers. Only segments with minimum length of 315 consecutive beats with valid RR and QT intervals were considered in the subsequent analysis. Longer segments were carved up respecting the minimum length admitted. 29 segments from POLI/MEDLAV database and 135 segments from Politecnico $\mathrm{Ca}^{\prime}$ Granda database were obtained, with a mean length of 415 and 402 beats (approximately 292.46 and 329.24 seconds), respectively.

\section{Results and discussion}

\subsection{Simulated data}

The chart in Figure 3 presents the errors across tests A, $\mathrm{B}$ and $\mathrm{C}$ in each frequency band using order 18. The corresponding mean and standard deviation $(S t d)$ of the errors can be found in Table 1 and the results were similar for all model orders admitted. More than half of the errors obtained in the tests $\mathrm{A}, \mathrm{B}$ and $\mathrm{C}$ were lower than $5 \%$ in all frequency bands and, in mean, these errors were lower than $1 \%$. Using higher sampling frequency or/and RR and QT intervals measured over artificial ECG (tests D, E and F), the errors did not differ relevantly from the previous ones (<1\%, except in one case), confirming the adequateness of the automatic detector presented in [3] for these studies. The increased error found on HF band $(4.19 \%)$ with sampling frequency 500 $\mathrm{Hz}$ reduces to $1.62 \%$ when tests $\mathrm{A} 1$ were excluded. This effect is due to the very low HF power of RR1 series (as illustrated in Fig. 2) and, consequently of QT in tests A1, resulting in a small absolute error on the estimated PSD measures holding a high percent importance.

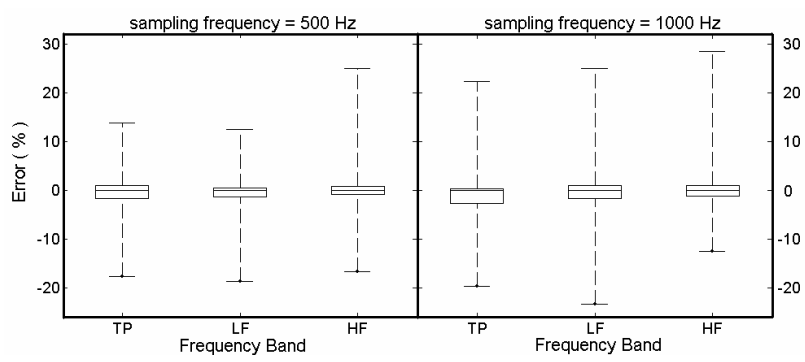

Figure 3. Box and whisker plots for the errors in the estimation of the QTV fraction uncorrelated to RR, $S_{Q T / W_{Q T}}(\omega)$, (test data A, B and C, order 18).

\subsection{Real data}

The normalised autocorrelation of the $\mathrm{W}_{\mathrm{QT}}$ series was not significantly different from zero for at least one of the studied orders in each segment, but in 4 of the segments we did not obtain a white noise $\mathrm{W}_{\mathrm{RR}}$ residue for any of the tested orders. The cross-correlation between $\mathrm{W}_{\mathrm{RR}}$ and $\mathrm{W}_{\mathrm{QT}}$ was not significantly different from zero in all cases. This allowed us to obtain adequate models for 28 out of 29 segments of POLI/MEDLAV and 132 out of 135 of Politecnico $\mathrm{Ca}^{\prime}$ Granda database. The method was also applied to the RR and RT series in the same segments.

The values found for the ratios (\%) between the measures on uncorrelated fraction and total QTV spectrum were very high, as illustrated in Figure 4 for order 18. The fraction uncorrelated with HRV was found to be higher than $40 \%$ in $98 \%$ of the segments for TP and HF band and $89 \%$ for LF. These results were similar for all model orders and suggest that other factors rather than RR could drive an important part of QTV.

As expected, the estimated power measures for RTapex variability were typically lower than for QTV. For order 18, the RT variability fraction uncorrelated with RR was higher than $40 \%$ in $100 \%$ of the segments for TP, but only $85 \%$ for HF band and $93 \%$ for LF. Very different values for the ratios can be found for the same segment considering QT or RTapex variability, suggesting different relations with RR. The variability present in the terminal part of the repolarization ( $T$ wave) contains both components correlated and uncorrelated with HRV.

It is worthwhile to remark that in this study we aimed to es timate the fraction of QTV that is not correlated with HRV. The uncorrelation between that part of QTV and HRV does not imply that there is not any physiological dependence between them. 
Table 1. Mean and standard deviation of errors on QTV fraction not correlated with RR, using simulated data (order 18).

\begin{tabular}{ccccc}
\cline { 2 - 4 } & \multicolumn{2}{c}{ Tests A1, B2, C2, A2, B2 and C2 } & \multicolumn{2}{c}{ Tests D1, E1, F1, D2, E2 and F2 } \\
\hline Sampling frequency & $\begin{array}{c}500 \mathrm{~Hz} \\
\text { Mean } \pm \text { Std }(\%)\end{array}$ & $\begin{array}{c}1000 \mathrm{~Hz} \\
\text { Mean } \pm \text { Std }(\%)\end{array}$ & $\begin{array}{c}500 \mathrm{~Hz} \\
\text { Mean } \pm \text { Std }(\%)\end{array}$ & $\begin{array}{c}1000 \mathrm{~Hz} \\
\text { Mean } \pm \operatorname{Std}(\%)\end{array}$ \\
\hline TP & $-0.38 \pm 4.56$ & $-0.78 \pm 5.05$ & $0.76 \pm 4.79$ & $-0.76 \pm 5.03$ \\
LF & $-0.51 \pm 3.96$ & $-0.11 \pm 5.61$ & $0.78 \pm 5.19$ & $-0.16 \pm 5.59$ \\
HF & $0.59 \pm 5.63$ & $0.82 \pm 6.07$ & $4.19 \pm 8.53$ & $0.86 \pm 6.08$ \\
\hline
\end{tabular}

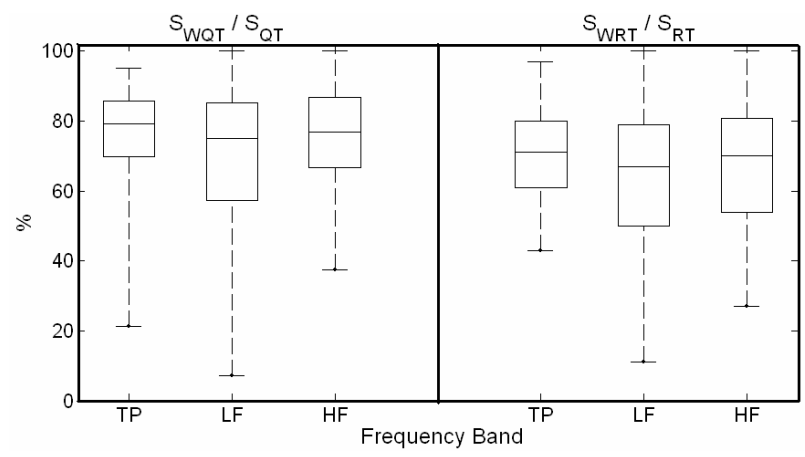

Figure 4. Box and whisker plots of the ratios between the measures of $\mathrm{S}_{\mathrm{QT} / \mathrm{WQT}}$ and $\mathrm{S}_{\mathrm{QT}}$ (left panel) and of $\mathrm{S}_{\mathrm{RT} / \mathrm{WRT}}$ and $\mathrm{S}_{\mathrm{RT}}$ (right panel) in real data (order 18).

Also the restriction of the same order for all the polynomials in the model is an artificial simplification. A strategy for order selection in each part of the model should be included by using a priori information and a goodness-of-fit measure, like Akaike's Information Theoretic Criteria.

\section{Concluding remarks}

The study of short term RR and QT relations, in particular the quantification of the QTV fraction uncorrelated with the HRV, is a complex and challenging problem. In this work, we used an approach analogous to the proposed by Porta et al [1] for the RT interval variability, by applying a linear low order model to assess QTV and HRV relations. The results point out that an important part of QTV (more than 40\%) is not driven by RR. The automated method presented in [3] has been shown (Table 1) adequate to quantify the QTV introducing errors much lower than ratio values found.

As a matter of fact, the results obtained are limited by the use of the same order for all the polynomials in the model and in the future a strategy for order selection in each part of the model should be included. Reducing the errors associated to the modeling and quantifying the effects of the residual correlations are important steps to improve the robustness of the proposed method.

An extension of this model by means of incorporating additional information should be considered to achieve better characterization of sources non-correlated with RR. Identification and interpretation of these phenomena is the driving force for future studies.

\section{Acknowledgements}

The first author acknowledges the grant SFRH / BD / 5484 / 2001 supported by FCT and ESF (III CSF). This work was also supported by the integrated action HP2001-0031 / CRUP-E26/02 and projects TIC20012167-C02-02 from MCYT/FEDER and P075/2001 from CONSID-DGA (Spain).

\section{References}

[1] Porta A, Baselli G, Caiani E, Malliani A, Lombardi F, Cerutti S. Quantifying electrocardiogram RT-RR variability interactions. Med. Biol. Eng. Comp. 1998; 36: $27-34$.

[2] Pueyo E, Smetana P, Hnatkoval K, LagunaP, Malik M. Time for QT Adaptation to RR Changes and Relation to Arrhythmic Mortality Reduction in Amiodarone-treated Patients. Computers in Cardiology 2002; 29: 565-568.

[3] Martínez JP, Almeida R, Olmos S, Rocha AP, Laguna P. A wavelet-based ECG delineator: Evaluation on standard databases. IEEE Trans. on Biomed. Eng., to appear.

[4] Mateo J, Laguna P. Improved Heart Rate Variability Signal Analysis from the Beat Occurrence Times According to the IPFM Model Heart Timing Signal. IEEE Trans. on Biom. Eng. 2000; 47: 985-996.

[5] Goodwin GC, Payne RL. Dynamic System Identification, Experimental Design and Data Analysis.1977; Academic Press.

[6] Pinciroli F, Pozzi G, Rossi R, Piovosi M, Capo A, Olivieri R, Della Torre M. A respiration-related EKG database. Computers in Cardiology 1988; 15: 477-480.

[7] Mateo J, Laguna P. Analysis of Heart Rate Variability in the Presence of Ectopic Beats Using the Heart Timing Signal. IEEE Trans. on Biome. Eng. 2003; 50: 334-342.

Address for correspondence:

Rute Almeida.

Faculdade de Ciências da Universidade do Porto

Departamento de Matemática Aplicada

Rua do Campo Alegre, 687; 4169-007, Porto, Portugal.

E-mail address: rbalmeid@fc.up.pt. 